\title{
Anxiety disorders and accelerated cellular ageing
}

Josine E. Verhoeven, Dóra Révész, Patricia van Oppen, Elissa S. Epel, Owen M. Wolkowitz and Brenda W. J. H. Penninx

\section{Background}

Anxiety disorders increase the risk of onset of several ageing-related somatic conditions, which might be the consequence of accelerated cellular ageing.

\section{Aims}

To examine the association between anxiety status and leukocyte telomere length (LTL) as an indicator of cellular ageing.

\section{Method}

Data are from individuals with current $(n=1283)$ and remitted $(n=459)$ anxiety disorder, and controls $(n=582)$ with no psychiatric disorder from the Netherlands Study of Depression and Anxiety. We determined DSM-IV anxiety diagnoses and clinical characteristics by structured psychiatric interviews and self-report questionnaires; LTL was assessed using quantitative polymerase chain reaction and converted into base pairs (bp).

\section{Results}

Patients in the current anxiety group $(b p=5431)$ had significantly shorter LTL compared with the control group ( $\mathrm{bp}=5506, P=0.01$ ) and the remitted anxiety group ( $\mathrm{bp}=$ 5499, $P=0.03$ ) in analyses adjusted for sociodemographics, health and lifestyle. The remitted anxiety group did not differ from the control group $(P=0.84)$, however, time since remission was positively related with $\mathrm{LTL}$. Furthermore, anxiety severity scores were associated with LTL in the whole sample, in line with a dose-response association.

\section{Conclusions}

Patients with current - but not remitted - anxiety disorder had shorter telomere length, suggesting a process of accelerated cellular ageing, which in part may be reversible after remission.

\section{Declaration of interest}

E.E. is a cofounder of Telomere Diagnostics, Inc., a telomere measurement company.

\section{Copyright and usage}

(c) The Royal College of Psychiatrists 2015.
Anxiety disorders not only interfere with a person's daily life by adversely having an impact on functioning and quality of life, ${ }^{1}$ but also by decreasing somatic health. ${ }^{2}$ Various longitudinal studies have confirmed that anxiety is a risk factor for several ageing-related somatic conditions including coronary heart disease, ${ }^{3}$ diabetes ${ }^{4}$ and disability, ${ }^{5}$ as well as for overall mortality. ${ }^{6}$ These increased risks remain even when controlling for lifestyle factors such as smoking, alcohol intake and physical activity, which suggests the involvement of underlying biological processes. Anxiety disorders are considered to be chronic and stressful conditions ${ }^{7}$ known to be accompanied by dysregulations of the major bodily stress systems including the hypothalamicpituitary-adrenal (HPA) axis, ${ }^{8}$ the autonomic nervous system and the immune system. ${ }^{10}$ It has been hypothesised that these stress-induced dysregulations result in accelerated cell ageing, ${ }^{11}$ which may partly account for the decreased somatic health in people with anxiety disorder.

Cellular ageing can be indexed by the length of telomeres, which are specialised nucleic acid-protein complexes that cap the ends of linear DNA and protect DNA from damage. Because of the 'end-replication problem', the final part of the telomere fails to be replicated during every cell division, causing telomeres to become progressively shorter. When telomeres reach a critically short length, cells become susceptible to senescence or apoptosis. ${ }^{12}$ Telomere shortening is counteracted by the cellular enzyme telomerase that adds telomeric DNA, thus preserving telomere length and healthy cell function. Accelerated shortening may be the consequence of disturbances of stress systems such as the HPA axis ${ }^{13}$ and the immune system. ${ }^{14}$ These dysregulations possibly contribute to telomere shortening by increasing oxidative stress, which in turn damages telomeres. ${ }^{14}$ Shortened telomeres have been shown to be a prognostic marker of various ageingrelated conditions such as cardiovascular disease, ${ }^{15}$ obesity, ${ }^{16}$ diabetes, ${ }^{17}$ cancer, ${ }^{18}$ cognitive decline ${ }^{19}$ and of earlier mortality. ${ }^{20}$
Although some recent studies have suggested patterns of accelerated cellular ageing among patients with depression ${ }^{21-24}$ research into cellular ageing in patients with anxiety disorders has been very limited up until now. One study by Kananen et $a l^{25}$ found shorter telomere length in patients with panic disorder, generalised anxiety disorder (GAD), social phobia or agoraphobia, but only in an older subgroup of the patients with anxiety disorder ( $>48$ years old) compared with a similar-aged control group, and not in the whole sample of 321 patients compared with 653 controls. Hoen et $a l^{26}$ found that 108 patients with an anxiety disorder had shorter telomere length than 970 non-anxious people at a 2-year follow-up period. Higher stress appraisals ${ }^{27}$ and high phobic anxiety ${ }^{28}$ were also found to be associated with shorter telomere length in non-psychiatric samples. In summary, there are only a few studies on the link between anxiety disorders and telomere length and results so far have been mixed. It thus remains unclear whether anxiety disorders are accompanied by accelerated cellular ageing. This paper examines the association between leukocyte telomere length (LTL) and the presence of the most common anxiety disorders: panic disorder with and without agoraphobia, GAD, social phobia and agoraphobia in a large adult sample $(n=2324)$ including people with current and remitted anxiety disorders and healthy controls. The large sample size allowed us to control for the most important confounding variables and to examine whether specific psychiatric characteristics, such as type of anxiety disorder, severity and symptom duration, have an impact on cellular ageing.

\section{Method}

\section{Study sample}

Data are from the baseline assessment of the Netherlands Study of Depression and Anxiety (NESDA), an ongoing longitudinal 
cohort study examining the course and consequences of depressive and anxiety disorders. The NESDA sample consists of 2981 participants between 18 and 65 years including people with a current or remitted diagnosis of a depressive and/or anxiety disorder (74\%) and healthy controls (26\%). To represent various settings and stages of psychopathology, participants with depression and anxiety disorders were recruited at three different locations in The Netherlands in different settings: the community, primary care and specialised mental healthcare settings. People with insufficient command of the Dutch language or a primary clinical diagnosis of other severe psychiatric conditions, such as bipolar disorder, obsessive-compulsive disorder (OCD), posttraumatic stress disorder (PTSD), severe substance use disorder or a psychotic disorder, as reported by themselves or their mental health practitioner, were excluded. Participants were recruited between September 2004 and February 2007. The study was approved by the ethical review board of participating centres, and all participants signed informed consent. Participants in NESDA were assessed during a $4 \mathrm{~h}$ clinic visit. The population and methods of the NESDA study have been described in more detail elsewhere. ${ }^{29}$

For the current study, three groups were created: a control group, a group of participants with remitted anxiety disorders and a group of participants with current anxiety disorders including panic disorder with and without agoraphobia, social phobia, GAD and agoraphobia, thereby excluding 657 participants who did not meet the criteria of one of the three groups. A total of 37 participants were subsequently excluded from analyses because of missing telomere length data, leaving 2324 individuals. Individuals in the control group $(n=582)$ were defined as having no lifetime history of anxiety or depressive disorders as assessed by the DSM-IV Composite International Diagnostic Interview (CIDI, version 2.1), ${ }^{30}$ and an anxiety severity score below 11 on Beck's Anxiety Inventory (BAI). ${ }^{31}$ Participants in the current anxiety disorder group $(n=1283)$ had one or more CIDI-diagnosed anxiety disorders (747 had one anxiety diagnosis, 404 had two diagnoses and 132 had three comorbid anxiety disorders) in the past 6 months. The participants in the remitted anxiety disorder group $(n=459)$ had an anxiety disorder previously but not in the past 6 months according to the CIDI.

\section{Measurements}

\section{Telomere length}

Fasting blood was drawn from participants in the morning between $08.30 \mathrm{~h}$ and $09.30 \mathrm{~h}$ and blood samples were stored in a $-80^{\circ} \mathrm{C}$ freezer afterwards. Leukocyte telomere length (LTL) was determined at the laboratory of Telomere Diagnostics, Inc. (Menlo Park, California, USA), using quantitative polymerase chain reaction ( $\mathrm{qPCR})$, adapted from the published original method by Cawthon et al. ${ }^{32}$ Telomere sequence copy number in each patient's sample ( $\mathrm{T}$ ) was compared with a single-copy gene copy number (S), relative to a reference sample. The resulting $\mathrm{T} / \mathrm{S}$ ratio is proportional to mean LTL. ${ }^{32,33}$ The detailed method is described elsewhere. $^{24}$

To compare T/S ratios to telomere restriction fragments (TRF) reported by studies using Southern blot analysis, we used the following steps to derive a conversion formula. Lin et $a l^{34}$ used a formula of base pairs (bp) $=3274+2413 \times \mathrm{T} / \mathrm{S}$ based on comparison of T/S ratios and TRF analysis of a series of genomic DNA samples from the human fibroblast cell line IMR90. Comparison of the T/S ratios of eight quality control DNA samples from the Telome Health Inc. laboratory that were included on each PCR run, generated the following formula: $\mathrm{T} / \mathrm{S}_{\left(\text {Lin } e t a l^{34}\right)}=\left(\mathrm{T} / \mathrm{S}_{(\text {TelomereDiagnostics })}-0.0545\right) / 1.16$. Therefore, the final formula we used to convert $\mathrm{T} / \mathrm{S}$ ratios to $\mathrm{bp}$ is:
$\mathrm{bp}=3274+2413((\mathrm{~T} / \mathrm{S}-0.0545) / 1.16)$. The reliability of the assay was adequate: eight included quality control DNA samples on each PCR run illustrated a small intra-assay coefficient of variation of $5.1 \%$ and the interassay coefficient of variation was also sufficiently low $(4.6 \%)$.

\section{Psychiatric characteristics}

Four severity measures were included, corresponding with the anxiety disorders in this study: (a) panic disorder arousal symptoms were assessed by the 21 -item $\mathrm{BAI}^{31}{ }^{31}$ (b) social phobic symptoms were measured with the five-item social phobia subscale of the Fear Questionnaire; ${ }^{35}$ (c) the five-item agoraphobia subscale of the Fear Questionnaire assessed agoraphobic symptoms; and (d) GAD's worrying and rumination were assessed with the Penn State Worry Questionnaire (PSWQ). ${ }^{36}$ Anxiety duration in recent years was assessed by the Life Chart interview (LCI), ${ }^{37}$ which uses a calendar method to assess the number of months in which anxious symptoms and avoidance were present during the past 4 years. The CIDI also assessed comorbid major depressive disorder (MDD), which was considered present when diagnosed in the past 6 months. Current use of psychoactive medication was measured by inspection of the active component or medication brand, the dose and frequency of intake from the label on the containers brought in and was categorised using the World Health Organization Anatomical Therapeutic Chemical (ATC) classification ${ }^{38}$ into antidepressants (tricyclic antidepressants (N06AA), selective serotonin reuptake inhibitors (N06AB) and other antidepressants (N06AF, N06AG, N06AX)) and benzodiazepines (N03Ae, N05BA, N05CD, N05CF).

\section{Covariates}

Gender, age and years of education were assessed during the interview. Body mass index (BMI) was calculated as measured weight divided by height-squared and divided into underweight (<18.5), normal (18.5-24.9), overweight (25.0-30.0) and obese $(>30.0)$. Alcohol consumption was categorised as non-drinker, moderate drinker (female $<14$ and male $<21$ drinks/week) or heavy drinker (female $\geqslant 14$ and male $\geqslant 21$ drinks/week). Smoking status was categorised into current, former or never smoker. Physical activity was assessed using the International Physical Activity Questionnaire (IPAQ) ${ }^{39}$ and expressed as overall energy expenditure in metabolic equivalent total (MET)-minutes per week (MET level $\times$ minutes activity $\times$ events per week, see Ainsworth et $a l^{40}$ ). The number of current somatic diseases for which participants received medical treatment (i.e. heart disease, epilepsy, diabetes, osteoarthritis, stroke, cancer, chronic lung disease, thyroid disease, liver disease, intestinal disorders and ulcers) was counted.

\section{Statistical analyses}

Baseline characteristics were compared across anxiety disorder status (control, remitted and current anxiety disorder groups) using chi-squared statistics and analyses of variance (ANOVA). We used analyses of covariance (ANCOVA) to determine differences in LTL in the remitted and current anxiety disorder groups compared with each other and with the control group, controlling for all covariates. Within the remitted anxiety group we tested whether the time since remission (in years) was associated with LTL to test the hypothesis that LTL restores with a longer time since remission. Also, sensitivity analyses were performed in which participants with current and lifetime MDD were excluded to check whether a possible association is present in people with anxiety disorder only and not caused by comorbid MDD. The extra analyses were all fully adjusted. To examine the 
role of type of anxiety disorder, adjusted mean LTL of the control group was compared with each of the five disorder subgroups: panic disorder with agoraphobia, panic disorder without agoraphobia, GAD, social phobia and agoraphobia. Cohen's $d$ was determined as an effect size estimation for significant results. Finally, covariate-adjusted multiple linear regression analyses were used to analyse the association of anxiety characteristics with LTL both within the total sample and within the current anxiety sample. All analyses were conducted using SPSS version 20 for Windows.

\section{Results}

Table 1 shows baseline characteristics across anxiety status. The mean age of the total study sample $(n=2324)$ was 41.7 years (s.d. $=13.1$, range $18-65)$ and $66.7 \%$ were female. The remitted anxiety disorder group was slightly older than the two other groups, while the control group contained fewer women than both anxiety groups. The current anxiety disorder group were more often less educated, current smokers and non-drinkers or heavy drinkers, and both remitted and current anxiety disorder groups had more somatic diseases than controls. Table 2 shows that the three groups differed on all psychiatric characteristics with the current anxiety disorder group showing the most severe characteristics. These data demonstrate that people with anxiety disorders are in several ways different from non-psychiatric controls and suggest that this epidemiological study sample consists of people with anxiety disorder as actually seen in the community, but that controlling for these differences is insuperable.

LTL was normally distributed and on average $5463 \mathrm{bp}$ $($ s.d. $=614)$. It exhibited a significant negative correlation with

\begin{tabular}{|c|c|c|c|c|}
\hline & $\begin{array}{l}\text { Control group } \\
\quad(n=582)\end{array}$ & $\begin{array}{l}\text { Remitted anxiety } \\
\text { disorder group }(n=459)\end{array}$ & $\begin{array}{c}\text { Current anxiety } \\
\text { disorder group }(n=1283)\end{array}$ & $P$ \\
\hline \multicolumn{5}{|l|}{ Demographics } \\
\hline Age, mean (s.d.) & $41.7(14.8)$ & $43.6(12.7)$ & $41.3(12.4)$ & 0.003 \\
\hline Gender: female: \% & 60.5 & 71.5 & 67.9 & $<0.001$ \\
\hline Years of education, mean (s.d.) & $12.9(3.2)$ & $12.4(3.2)$ & $11.6(3.3)$ & $<0.001$ \\
\hline \multicolumn{5}{|l|}{ Health and lifestyle } \\
\hline \multicolumn{5}{|l|}{ Body mass index, $\%$} \\
\hline Underweight & 2.1 & 1.5 & 2.5 & \\
\hline Normal & 54.1 & 48.6 & 51.0 & 0.06 \\
\hline Overweight & 30.6 & 32.9 & 28.1 & \\
\hline Obese & 13.2 & 17.0 & 18.4 & \\
\hline \multicolumn{5}{|l|}{ Smoking status, \% } \\
\hline Never & 37.6 & 27.0 & 24.8 & \\
\hline Former & 35.6 & 39.2 & 29.6 & $<0.001$ \\
\hline Current & 26.8 & 33.8 & 45.6 & \\
\hline \multicolumn{5}{|l|}{ Alcohol status, \% } \\
\hline Non-drinker & 10.3 & 15.0 & 21.9 & \\
\hline Moderate drinker & 78.2 & 73.2 & 65.0 & $<0.001$ \\
\hline Heavy drinker & 11.5 & 11.8 & 13.1 & \\
\hline Physical activity, in 1000 MET-min a per week: mean (s.d.) & $3.8(3.0)$ & $3.6(2.8)$ & $3.6(3.0)$ & 0.33 \\
\hline Number of somatic diseases, mean (s.d.) & $0.5(0.8)$ & $0.6(0.9)$ & $0.7(0.9)$ & $<0.001$ \\
\hline
\end{tabular}

\begin{tabular}{|c|c|c|c|c|}
\hline & $\begin{array}{l}\text { Control group } \\
\quad(n=582)\end{array}$ & $\begin{array}{l}\text { Remitted anxiety } \\
\text { disorder group }(n=459)\end{array}$ & $\begin{array}{c}\text { Current anxiety } \\
\text { disorder group }(n=1283)\end{array}$ & $P^{\mathrm{a}}$ \\
\hline $\begin{array}{l}\text { Type of anxiety disorder, } n \\
\text { Agoraphobia } \\
\text { Panic disorder with agoraphobia } \\
\text { Panic disorder without agoraphobia } \\
\text { Social phobia } \\
\text { Generalised anxiety disorder }\end{array}$ & NA & $\begin{array}{r}68 \\
52 \\
99 \\
175 \\
193\end{array}$ & $\begin{array}{l}187 \\
414 \\
224 \\
652 \\
454\end{array}$ & NA \\
\hline Time since remission, years: mean (s.d.) & NA & $18.0(18.7)$ & NA & NA \\
\hline Number of anxiety disorders, mean (s.d.) & NA & NA & $1.5(0.7)$ & NA \\
\hline $\begin{array}{l}\text { Anxiety severity, mean (s.d.) } \\
\text { Anxiety arousal (Beck's Anxiety Inventory) } \\
\text { Social fear (subscale Fear Questionnaire) } \\
\text { Agoraphobia (subscale Fear Questionnaire) } \\
\text { Worrying (Penn State Worry Questionnaire) }\end{array}$ & $\begin{aligned} & 2.9(2.9) \\
& 5.2(5.1) \\
& 1.6(3.4) \\
& 18.4(9.0)\end{aligned}$ & $\begin{aligned} 8.9 & (7.3) \\
9.7 & (7.0) \\
4.1 & (5.7) \\
26.1 & (14.1)\end{aligned}$ & $\begin{array}{l}18.5(10.8) \\
15.9(9.2) \\
11.1(9.8) \\
31.3(16.8)\end{array}$ & $\begin{array}{l}<0.001 \\
<0.001 \\
<0.001 \\
<0.001\end{array}$ \\
\hline Duration, months with symptoms in past 4 years: mean (s.d.) & NA & $16.9(16.8)$ & $31.3(20.5)$ & $<0.001$ \\
\hline Comorbid major depressive disorder, \% & NA & 23.3 & 56.0 & $<0.001$ \\
\hline $\begin{array}{l}\text { Antidepressant use, \% } \\
\text { Tricyclic antidepressant } \\
\text { Selective serotonin reuptake inhibitor } \\
\text { Other antidepressant }\end{array}$ & $\begin{array}{l}0.2 \\
0.5 \\
0.2\end{array}$ & $\begin{array}{r}2.4 \\
16.3 \\
3.5\end{array}$ & $\begin{array}{r}4.4 \\
26.8 \\
9.1\end{array}$ & $\begin{array}{l}<0.001 \\
<0.001 \\
<0.001\end{array}$ \\
\hline Benzodiazepine use, \% & 0.5 & 3.9 & 13.1 & $<0.001$ \\
\hline
\end{tabular}


age $(r=-0.326, \quad P<0.001)$ that corresponded to a mean shortening rate of $14 \mathrm{bp} /$ year. This shortening rate is comparable with previously reported rates based on cross-sectional data ( $14 \mathrm{bp} /$ year by Cawthon et al, ${ }^{20} 20 \mathrm{bp} /$ year by Hartmann et al $\mathrm{l}^{22}$ and $15-17 \mathrm{bp} /$ year by Wikgren et $\left.a^{23}\right)$. Female participants had longer LTL than male participants $(F=14.23 ; P<0.001$, corrected for age). LTL was, besides age and gender, associated with weight (shorter LTL for being underweight, overweight or obese), smoking and drinking status (former and current smokers as well as heavy drinkers have shorter LTL) and number of somatic diseases (more diseases, shorter LTL), but not with education and physical activity.

Between-group analyses showed that the LTL of the control, remitted and current anxiety groups differed in unadjusted $(F(2,2321)=3.57, \quad P=0.028)$, sociodemographicadjusted $(F(2,2318=5.40, \quad P=0.005)$ and fully adjusted (sociodemographic, health and lifestyle) analyses $(F(2,2313=$ $4.22, P=0.015)$. Post hoc analyses showed that those in the current anxiety group had shorter LTL than healthy controls in unadjusted analyses (Table 3). Further, when adjusted for age, gender and education, LTL was significantly shorter for the current anxiety group $(\mathrm{bp}=5427)$ compared with the control (mean $\mathrm{bp}=5514$, $P=0.003)$ and the remitted anxiety group (bp $=5499$; $P=0.025)$. Differences remained significant in analyses fully adjusted for sociodemographic, health and lifestyle variables: the current anxiety group had on average $75 \mathrm{bp}$ shorter LTL compared with the control group $(P=0.01$, Cohen's $d=0.13)$ and $68 \mathrm{bp}$ shorter LTL $(P=0.03$, Cohen's $d=0.12)$ compared with the remitted group (see Table 3 for the $95 \%$ confidence intervals). The remitted anxiety group did not significantly differ from the control group $(P=0.84)$. However, within the remitted anxiety group, the time since remission was positively associated with LTL ( $\beta=0.107, P=0.024$, adjusted for all covariates). In further analysis we performed a median-split, resulting in a subgroup that remitted between 6 months to 9 years ago $(n=225)$ and a group that were remitted for 10 years or longer $(n=220)$. The first subgroup $(b p=5372)$ had significantly shorter LTL than the $10+$ years subgroup $(\mathrm{bp}=5529 ; P=0.022$; Cohen's $d=0.22)$, suggesting that the cellular ageing process is in part reversible.

In contrast, in an earlier study in the same cohort we found that individuals who had had depression earlier in life but did not currently meet the MDD diagnosis had shorter telomeres than controls. ${ }^{24}$ Both the remitted patient groups differed from each other in terms of subthreshold symptomatology and length of remission: remitted patients with MDD scored 18.0 (s.d.=10.2) on the Inventory of Depressive Symptoms, ${ }^{24}$ which is still indicative of 'mild symptomatology'; ${ }^{41}$ whereas the remitted patients with anxiety disorder scored 8.9 (s.d. $=7.3$ ) on BAI, which is below the clinically relevant cut-off score of $11 .^{42}$ Moreover, the average time to remission in the remitted patients with MDD was 5.2 years $($ s.d. $=6.2)$, whereas it was much longer in the remitted anxiety group: 18.0 years $($ s.d. $=18.7)$ years.
To test whether the associations found in the present study were not caused by comorbid depressive conditions, sensitivity analyses were performed in which participants with current MDD were excluded. These analyses showed that the 564 participants with current anxiety disorders (but no current MDD) still had significantly shorter LTL than controls $(P=0.021$; Cohen's $d=0.14)$ and marginally significantly shorter telomeres than the participants with remitted anxiety disorders ( $P=0.078$; both adjusted for all covariates), indicating that the association is truly present in those with anxiety disorders and not just caused by comorbid depressive conditions. Even when excluding lifetime MDD diagnoses, 268 participants with current anxiety disorders had shorter LTL compared with the controls $(P=0.024)$, but not compared with those participants in the remitted anxiety group $(P=0.273)$.

To examine the role of type of disorder, mean LTLs for the different anxiety disorder subgroups were each compared with the control group (Fig. 1). To reflect high comorbidity rates, patients with comorbid disorders were included in each anxiety disorder subgroup for which they met the diagnosis. Patients with panic disorder with agoraphobia $(b p=5428, P=0.03$, Cohen's $d=0.14$ ), social phobia ( $\mathrm{bp}=5443, P=0.04$, Cohen's $d=0.12$ ) and GAD ( $\mathrm{bp}=5404, P=0.002$, Cohen's $d=0.18$ ) had significantly shorter LTL than the control group. Patients with agoraphobia (bp $=5497, P=0.91)$ and panic disorder without agoraphobia $(\mathrm{bp}=5458, P=0.19)$, however, did not differ from controls.

Subsequently, the association between several anxiety characteristics and LTL was examined in the total sample as well as within the 1283 participants in the current anxiety disorder group (Table 4). Within the total sample, severity of anxiety arousal symptoms $(\beta=-0.056, P=0.007)$, social phobic symptoms $(\beta=-0.043, P=0.03)$ and worrying $(\beta=-0.041, P=0.04)$ were associated with shorter LTL. A higher number of current anxiety disorder diagnoses was also significantly associated with shorter LTL in the total sample $(\beta=-0.047, P=0.020)$, confirming the difference between people with and without a current anxiety disorder. No LTL-associations were found for symptom duration in the past 4 years $(P=0.14)$, comorbid MDD $(P=0.54)$ or the use of psychopharmacological medications (Table 4). Also, no anxiety characteristics were associated with LTL within the current anxiety disorder group by itself, suggesting that differences in LTL were only apparent when using the entire spectrum of participants in the healthy control and anxiety groups, but did not further differentiate within the current anxiety group.

\section{Discussion}

\section{Main findings}

This large-scale study demonstrated that patients with a current anxiety disorder on average had shorter LTL than non-psychiatric controls and patients with remitted anxiety disorder. Although

\begin{tabular}{|c|c|c|c|c|c|c|c|c|c|}
\hline & \multicolumn{3}{|c|}{ LTL, mean (s.e.) } & \multirow{2}{*}{\multicolumn{2}{|c|}{$\begin{array}{l}\text { Remitted anxiety disorder } \\
\text { v. control group }\end{array}$}} & \multirow{2}{*}{\multicolumn{2}{|c|}{$\begin{array}{c}\text { Current anxiety disorder } \\
\text { v. control group }\end{array}$}} & \multirow{2}{*}{\multicolumn{2}{|c|}{$\begin{array}{l}\text { Current anxiety disorder } \\
\text { v. remitted anxiety } \\
\text { disorder group }\end{array}$}} \\
\hline & \multirow{2}{*}{$\begin{array}{l}\text { Control } \\
\text { group } \\
(n=582)\end{array}$} & \multirow{2}{*}{$\begin{array}{l}\text { Remitted anxiety } \\
\text { disorder group } \\
(n=459)\end{array}$} & \multirow{2}{*}{$\begin{array}{l}\text { Current anxiety } \\
\text { disorder group } \\
\quad(n=1283)\end{array}$} & & & & & & \\
\hline & & & & $95 \% \mathrm{Cl}$ & $P$ & $95 \% \mathrm{Cl}$ & $P$ & $95 \% \mathrm{Cl}$ & $P$ \\
\hline Unadjusted & $5516(26)$ & $5474(28)$ & $5436(17)$ & -32.4 to 117.8 & 0.27 & 20.8 to 141.0 & 0.008 & -27.2 to 103.6 & 0.25 \\
\hline Basic adjustment ${ }^{a}$ & $5514(24)$ & $5499(27)$ & $5427(16)$ & -86.9 to 55.9 & 0.67 & 29.1 to 144.5 & 0.003 & 9.1 to 133.6 & 0.03 \\
\hline Full adjustment ${ }^{\mathrm{b}}$ & $5506(25)$ & $5499(27)$ & $5431(16)$ & -79.0 to 64.1 & 0.84 & 16.8 to 134.0 & 0.01 & 5.6 to 130.4 & 0.03 \\
\hline
\end{tabular}




\begin{tabular}{|c|c|c|c|c|}
\hline & \multicolumn{2}{|c|}{ Total sample $(n=2324)$} & \multicolumn{2}{|c|}{ Current anxiety disorder group $(n=1283)$} \\
\hline & $\beta$ & $P$ & $\beta$ & $P$ \\
\hline \multicolumn{5}{|l|}{ Anxiety severity } \\
\hline Anxiety arousal (Beck's Anxiety Inventory) & -0.056 & $<0.01$ & -0.029 & 0.30 \\
\hline Social fear (subscale Fear Questionnaire) & -0.043 & 0.03 & -0.029 & 0.28 \\
\hline Agoraphobia (subscale Fear Questionnaire) & -0.021 & 0.31 & 0.011 & 0.70 \\
\hline Worrying (Penn State Worry Questionnaire) & -0.041 & 0.04 & -0.002 & 0.95 \\
\hline Duration (months with anxiety symptoms in past 4 years) & -0.029 & 0.14 & -0.010 & 0.73 \\
\hline Number of current anxiety disorders & -0.047 & 0.02 & -0.001 & 0.98 \\
\hline Comorbid major depressive disorder & -0.012 & 0.54 & 0.016 & 0.54 \\
\hline \multicolumn{5}{|l|}{ Antidepressant use } \\
\hline Tricyclic antidepressant & -0.006 & 0.76 & 0.011 & 0.67 \\
\hline Selective serotonin reupake inhibitor & -0.033 & 0.10 & -0.009 & 0.73 \\
\hline Other antidepressant & -0.036 & 0.07 & -0.013 & 0.64 \\
\hline Benzodiazepine use & -0.007 & 0.73 & -0.002 & 0.94 \\
\hline
\end{tabular}

effect sizes were modest, the difference may indicate 3-5 years of accelerated ageing for the current anxiety group, based on the estimated mean telomere shortening rate of 14-20 bp/year as found in our and other studies. ${ }^{20,22,23}$ The observed associations remained significant after controlling for lifestyle and health variables suggesting that the shorter telomeres were not simply because of a unhealthy lifestyle or physical illnesses within the anxiety group.

Within the current anxiety disorder group, we showed that type of anxiety disorder further differentiated across disorders: patients with panic disorder with agoraphobia, social phobia and GAD, but not panic disorder without agoraphobia and agoraphobia without panic disorder, had significantly shorter LTL than the control group. The differences in LTL for the anxiety subgroups corresponded with an estimated 3.5-5 years (social phobia), 4-6 years (panic disorder with agoraphobia) and 5.5-8 years (GAD) of accelerated ageing compared with the control group. Based on descriptive information (data not shown) it appeared that the two anxiety disorders that did not differ from controls in terms of LTL, showed less severe characteristics compared with other anxiety disorder subgroups. For instance, participants with agoraphobia and panic disorder without agoraphobia tended to have a smaller number of comorbid anxiety disorders, less often comorbid depression and/or slightly lower anxiety severity scores than the other three anxiety subgroups. Consequently, the fact that participants with agoraphobia and panic disorder without agoraphobia reported generally milder symptomatology might explain why LTL was less

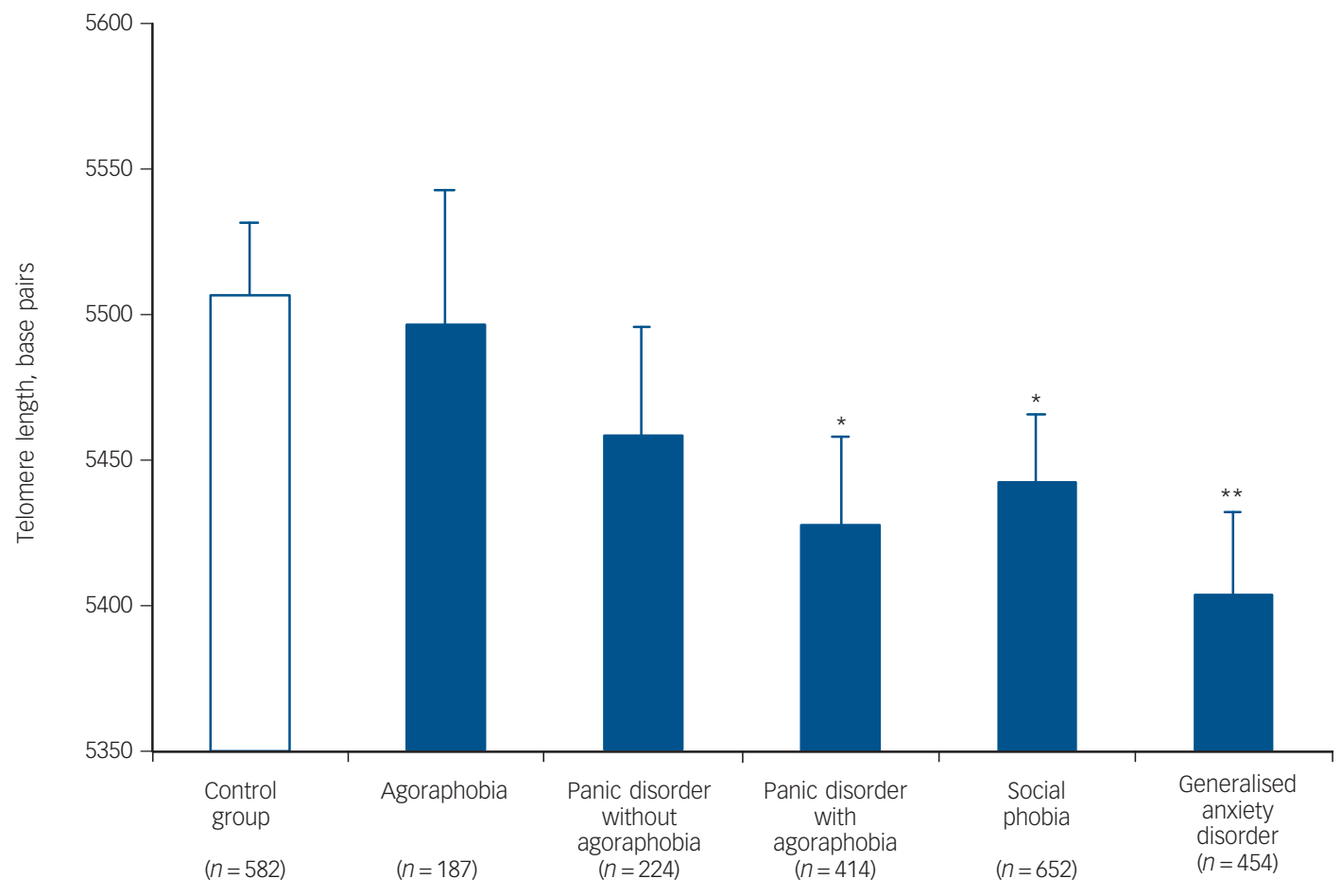

Fig. 1 Mean leukocyte telomere length (with s.e.) across various anxiety disorder subgroups $v$. the control group.

Patients with comorbid anxiety disorders appear in more than one disorder subgroup. All analyses are adjusted for age, gender, education, body mass index, smoking, alcohol, 
significantly decreased in these subgroups than in, for example. patients with panic disorder with agoraphobia, social phobia and GAD.

Our results suggest that telomere shortening might in part be reversible since the LTL difference with controls was not present in the group with remitted anxiety disorder. Furthermore, the time since remission was positively associated with LTL within the remitted anxiety group and participants with a remission time under 10 years had shorter telomeres than those who were remitted for 10 years or longer. An earlier study in the same cohort showed that patients with remitted MDD had shorter LTL than controls, suggesting that depression does leave a 'biological scar. ${ }^{24}$ But alternatively, the fact that there was a difference in LTL between the patients with remitted MDD compared with controls, whereas it did not differ in the patients with remitted anxiety disorder, might have been the result of the relatively higher levels of subthreshold symptoms and shorter remission time found in the remitted MDD group.

We further found that anxiety severity scores (anxiety arousal, social fear and worrying) were negatively associated with LTL in the total study sample. However, these variables were not associated with LTL within the current anxiety group, suggesting a dose-response effect across the entire spectrum of healthy controls and participants with anxiety but no further differentiations within the current anxiety group. In line with this, the number of anxiety disorders was also associated only in the total study sample. An explanation for this is that the overall effect of diagnosis status is stronger than that of a dose-response relationship within the participants with anxiety. Alternatively, the severity and duration measures used in our study may not fully represent the 'anxiety dose' component in patients. For instance, the anxiety severity measurements used were reflectors of symptom severity over the past week, but not over a much longer period and may therefore not be the best representation of general anxiety severity in patients. Duration of anxiety symptoms was not associated with LTL in this study. This might be as a result of the fact that generally most patients with anxiety disorder reported rather long symptom duration and therefore the range in this variable may not have been enough to illustrate associations with LTL. Comorbid MDD was not associated with LTL either, suggesting that the effects of anxiety disorder status on LTL were independent of MDD diagnosis, which was moreover confirmed by sensitivity analyses that showed that LTL of participants with anxiety disorder still differed from controls when we excluded patients with current and lifetime MDD. We did not find an association between psychopharmological medication and LTL, but more specific relationships between duration, type and dose of medication remain to be investigated.

\section{Possible mechanisms}

Our results align with the general tendency of the limited research into telomere length and anxiety disorders up until now: previous studies found anxiety disorders to be prospectively related to reduced telomere length, ${ }^{26}$ and telomere length to be shorter in an older subgroup of participants with anxiety disorder compared with a similar-aged control group. ${ }^{25}$ The observed shorter LTL in this study might be the consequence of several disturbances found in people with anxiety disorders, such as dysregulations in the major bodily stress systems such as the HPA axis ${ }^{8}$ and the immune system. ${ }^{10}$ Dysregulations of these stress systems could contribute to telomere shortening in patients with anxiety disorders. ${ }^{11,43}$ In line with this, several in vitro and in vivo studies found increased cortisol, ${ }^{44}$ oxidative stress ${ }^{45}$ and proinflammatory cytokines ${ }^{46}$ to be associated with shorter telomere length. However, the exact biological mechanisms that mediate the relationship between certain anxiety disorders and telomere shortening, as well as the direction of the link, remain to be further explored.

\section{Strengths and limitations}

The major strengths of the present study are the large sample size, the inclusion of participants with well-characterised current and remitted anxiety disorder diagnoses as well as healthy controls, the wide age range and the assessment of important covariates such as somatic health and lifestyle variables. However, some limitations of the present study should also be noted. First, the associations between anxiety disorder status and LTL were of rather small effect size. However, our effect sizes (Cohen's $d=0.12-0.22$ ) are not very different from those described in recent studies on pathophysiological mechanisms, for example, increased inflammatory markers in patients with anxiety disorder (Cohen's $d=0.15-0.19),{ }^{10}$ and decreased brain-derived neurotrophic factor (Cohen's $d=0.15-0.23)^{47}$ and increased cortisol (Cohen's $d=0.15-0.25)^{48}$ across MDD status. Second, as stress arousal is inherent in people with anxiety disorder, it is possible that the association between anxiety disorder status and telomere length is - partly - mediated by exposure to stressful events and experienced stress. By controlling for stress, the sample might no longer reflect ecologically valid anxiety disorders. It is, however, important to note that the observed telomere shortening might not be specific to anxiety disorders. Third, the sample studied may not fully represent the population with anxiety disorders at large, since for example PTSD and OCD, which can be comorbid with the anxiety disorders in our sample, were exclusion criteria. Fourth, our study design was cross-sectional, which might undermine the complexity of telomere length regulating mechanisms, and which does not allow one to draw conclusions regarding causality. Even though we looked at patients with current and remitted anxiety disorders, suggestive of a temporal effect, this study is solely a reflection of the current state of different participants. Since longitudinal studies show complex telomere length dynamics with both shortening as well as lengthening of telomeres over time, ${ }^{49-52}$ future studies should explore the relationship longitudinally. It should also be noted that our variable base pairs is an estimate rather than a directly measured variable. Also, as in nearly all studies, we used leukocytes for telomere length measurement, which is a validated, non-invasive and often-used indicator for cellular ageing. It would, however, be worthwhile to examine cellular ageing processes in other tissues. Finally, telomerase activity was not measured in the current study, but information regarding telomere repair and maintenance would be of great value in future research, since this would give us a more dynamic picture of the telomere/telomerase system in affective disorders.

\section{Clinical implications and further research}

This large cohort study provides suggestive evidence that patients with anxiety disorders have shorter telomere length compared with healthy controls, suggesting a pathway of accelerated cellular ageing within the most common anxiety disorders. This might in turn explain the increased somatic health problems found in this population. As participants with remitted anxiety disorder resembled the healthy controls, in terms of telomere length, it is possible that this telomere shortening can be reversed, although the present data did not allow assessment of within-participant changes in telomere length. Lifestyle interventions such as increased physical activity have been shown to have a favourable impact on cellular ageing in a healthy population. ${ }^{53-55}$ 
Therefore, further studies should test whether such interventions could result not only in a reversal of psychiatric symptomatology but also in restoring cellular ageing and consequent somatic health.

Josine E. Verhoeven, MSC, Dóra Révész, MSC, Patricia van oppen, PhD, Department of Psychiatry and EMGO Institute for Health and Care Research, VU University Medical Center, Amsterdam, The Netherlands; Elissa S. Epel, PhD, Owen M. Wolkowitz, MD, Department of Psychiatry, University of California, San Francisco, School of Medicine, San Francisco, California, USA; Brenda W. J. H. Penninx, PhD, Department of Psychiatry and EMGO Institute for Health and Care Research, VU University Medical Center, Amsterdam, The Netherlands

Correspondence: Josine E. Verhoeven, PO Box 74077, 1070 BB Amsterdam, The Netherlands. Email: J.Verhoeven@ ggzingeest.nl

\section{Funding}

The infrastructure for the NESDA study (www.nesda.nl) is funded through the Geestkrach program of the Netherlands Organization for Health Research and Development (Zon-MW,
grant number 10-000-1002) and is supported by participating universities and mental health grant number 10-000-1002) and is supported by participating universities and mental health
care organizations (VU University Medical Center, GGZ inGeest, Arkin, Leiden University Medical Center, GGZ Rivierduinen, University Medical Center Groningen, Lentis, GGZ Friesland, GGZ Drenthe, Institute for Quality of Health Care (IQ Healthcare), Netherlands Institute for Health Services Research (NIVEL) and Netherlands Institute of Mental Health and Addiction (Trimbos). B.W.J.H.P. J.E.V., D.R and telomere length assaying were supported through a NWO-VICl grant (number 91811602).

\section{References}

1 Bijl RV, Ravelli A. Current and residual functional disability associated with psychopathology: findings from the Netherlands Mental Health Survey and Incidence Study (NEMESIS). Psychol Med 2000; 30: 657-68.

2 Roy-Byrne PP, Davidson KW, Kessler RC, Asmundson GJG, Goodwin RD, Kubzansky L, et al. Anxiety disorders and comorbid medical illness. Gen Hosp Psychiatry 2008; 30: 208-25.

3 Roest AM, Martens EJ, de JP, Denollet J. Anxiety and risk of incident coronary heart disease: a meta-analysis. J Am Coll Cardiol 2010; 56: 38-46.

4 Atlantis E, Vogelzangs N, Cashman K, Penninx BJWH. Common mental disorders associated with 2-year diabetes incidence: the Netherlands Study of Depression and Anxiety (NESDA). J Affect Disord 2012; 142 (Suppl): S30-5.

5 Brenes GA, Penninx BW, Judd PH, Rockwell E, Sewell DD, Wetherell JL. Anxiety, depression and disability across the lifespan. Aging Ment Health 2008; 12: 158-63.

6 Denollet J, Maas K, Knottnerus A, Keyzer JJ, Pop VJ. Anxiety predicted premature all-cause and cardiovascular death in a 10-year follow-up of middle-aged women. J Clin Epidemiol 2009; 62: 452-6.

7 Penninx BWJH, Nolen WA, Lamers F, Zitman FG, Smit JH, Spinhoven P, et al. Two-year course of depressive and anxiety disorders: results from the Netherlands Study of Depression and Anxiety (NESDA). J Affect Disord 2011; 133: $76-85$

8 Vreeburg S, Zitman F, van der Pelt J, de Rijk R, Verhagen J, van Dyck R, et al Anxiety disorder and HPA-axis activity: results from a large cohort study. Psychosom Med 2010; 72: 340-7.

9 Licht CM, de Geus EJ, van DR, Penninx BW. Association between anxiety disorders and heart rate variability in The Netherlands Study of Depression and Anxiety (NESDA). Psychosom Med 2009; 71: 508-18.

10 Vogelzangs N, Beekman ATF, de Jonge P, Penninx BWJH. Anxiety disorders and inflammation in a large adult cohort. Transl Psychiatry 2013; 3: e249.

11 Wolkowitz OM, Epel ES, Reus VI, Mellon SH. Depression gets old fast: do stress and depression accelerate cell aging? Depress Anxiety 2010; 27 327-38.

12 Blackburn EH. Switching and signaling at the telomere. Cell 2001; 106 661-73.

13 Tomiyama AJ, O'Donovan A, Lin J, Puterman E, Lazaro A, Chan J, et al. Does cellular aging relate to patterns of allostasis? An examination of basal and stress reactive HPA axis activity and telomere length. Physiol Behav 2012; 106: $40-5$.

14 De la Fuente $\mathrm{M}$, Miquel J. An update of the oxidation-inflammation theory of aging: the involvement of the immune system in oxi-inflamm-aging. Curr Pharm Des 2009; 15: 3003-26.
15 Fitzpatrick AL, Kronmal RA, Gardner JP, Psaty BM, Jenny NS, Tracy RP, et al. Leukocyte telomere length and cardiovascular disease in the cardiovascular health study. Am J Epidemiol 2007; 165: 14-21.

16 Valdes AM, Andrew T, Gardner JP, Kimura M, Oelsner E, Cherkas LF, et al. Obesity, cigarette smoking, and telomere length in women. Lancet 2005; 366: 662-4.

17 Sampson MJ, Winterbone MS, Hughes JC, Dozio N, Hughes DA. Monocyte telomere shortening and oxidative DNA damage in type 2 diabetes. Diabetes Care 2006; 29: 283-9.

18 Willeit $\mathrm{P}$, Willeit J, Mayr A, Weger S, Oberhollenzer F, Brandstatter A, et al. Telomere length and risk of incident cancer and cancer mortality. JAMA 2010; 304: 69-75

19 Martin-Ruiz C, Dickinson HO, Keys B, Rowan E, Kenny RA, von Zglinicki T. Telomere length predicts poststroke mortality, dementia, and cognitive decline. Ann Neurol 2006; 60: 174-80.

20 Cawthon RM, Smith KR, O'Brien E, Sivatchenko A, Kerber RA. Association between telomere length in blood and mortality in people aged 60 years or older. Lancet 2003; 361: 393-5

21 Simon NM, Smoller JW, MCNamara KL, Maser RS, Zalta AK, Pollack MH, et al. Telomere shortening and mood disorders: preliminary support for a chronic stress model of accelerated aging. Biol Psychiatry 2006; 60: 432-5.

22 Hartmann N, Boehner M, Groenen F, Kalb R. Telomere length of patients with major depression is shortened but independent from therapy and severity of the disease. Depress Anxiety 2010; 27: 1111-6.

23 Wikgren M, Maripuu M, Karlsson T, Nordfjall K, Bergdahl J, Hultdin J, et al. Short telomeres in depression and the general population are associated with a hypocortisolemic state. Biol Psychiatry 2012; 71: 294-300.

24 Verhoeven J, Révész D, Epel E, Lin J, Wolkowitz O, Penninx B. Major depressive disorder and accelerated cellular aging: results from a large psychiatric cohort study. Mol Psychiatry 2014; 19: 895-901.

25 Kananen L, Surakka I, Pirkola S, Suvisaari J, Lonnqvist J, Peltonen L, et al. Childhood adversities are associated with shorter telomere length at adult age both in individuals with an anxiety disorder and controls. PLOS One 2010; 5: e10826.

26 Hoen PW, Rosmalen JGM, Schoevers RA, Huzen J, van der Harst P, de Jonge $P$. Association between anxiety but not depressive disorders and leukocyte telomere length after 2 years of follow-up in a population-based sample. Psychol Med 2013; 43: 689-97.

27 O'Donovan A, Tomiyama AJ, Lin J, Puterman E, Adler NE, Kemeny M, et al. Stress appraisals and cellular aging: a key role for anticipatory threat in the relationship between psychological stress and telomere length. Brain Behav Immun 2012; 26: 573-9.

28 Okereke OI, Prescott J, Wong JYY, Han J, Rexrode KM, De Vivo I. High phobic anxiety is related to lower leukocyte telomere length in women. PLOS One 2012; 7: e40516.

29 Penninx BWJH, Beekman ATF, Smit JH, Zitman FG, Nolen WA, Spinhoven P, et al. The Netherlands Study of Depression and Anxiety (NESDA): rationale, objectives and methods. Int J Methods Psychiatr Res 2008; 17: 121-40.

30 World Health Organization. Composite International Diagnostic Interview (CIDI). Version 2.1. WHO, 1997

31 Beck AT, Epstein N, Brown G, Steer RA. An inventory for measuring clinical anxiety: psychometric properties. J Consult Clin Psychol 1988; 56: 893-7.

32 Cawthon RM. Telomere measurement by quantitative PCR. Nucleic Acids Res 2002; 30: e47.

33 Aviv A, Hunt SC, Lin J, Cao X, Kimura M, Blackburn E. Impartial comparative analysis of measurement of leukocyte telomere length/DNA content by Southern blots and qPCR. Nucleic Acids Res 2011; 39: e134.

34 Lin J, Epel E, Cheon J, Kroenke C, Sinclair E, Bigos M, et al. Analyses and comparisons of telomerase activity and telomere length in human $\mathrm{T}$ and B cells: insights for epidemiology of telomere maintenance. $J$ Immunol Methods 2010; 352: 71-80.

35 Marks IM, Mathews AM. Brief standard self-rating for phobic patients. Behav Res Ther 1979; 17: 263-7.

36 Meyer TJ, Miller ML, Metzger RL, Borkovec TD. Development and validation of the Penn State Worry Questionnaire. Behav Res Ther 1990; 28: 487-95.

37 Lyketsos CG, Nestadt G, Cwi J, Heithoff K. The life-chart interview: a standardized method to describe the course of psychopathology. Int $J$ Methods Psychiatr Res 1994; 4: 143-55.

38 World Health Organization Collaboration Centre for Drug Statistics Methodology. Anatomical Therapeutic Chemical (ATC) Classification System. World Health Organization Collaboration Centre for Drug Statistics Methodology, 2007.

39 Craig $\mathrm{CL}$, Marshall $\mathrm{AL}$, Sjostrom $\mathrm{M}$, Bauman $\mathrm{AE}$, Booth $\mathrm{ML}$, Ainsworth $\mathrm{BE}$, et al. International physical activity questionnaire: 12 -country reliability and validity. Med Sci Sports Exerc 2003; 35: 1381-95. 
40 Ainsworth BE, Haskell WL, Herrmann SD, Meckes N, Bassett DRJ, Tudor-Locke C, et al. 2011 compendium of physical activities: a second update of codes and MET values. Med Sci Sports Exerc 2011; 43: 1575-81.

41 Rush AJ, Trivedi MH, Ibrahim HM, Carmody TJ, Arnow B, Klein DN, et al. The 16-Item Quick Inventory of Depressive Symptomatology (QIDS), clinician rating (QIDS-C), and self-report (QIDS-SR): a psychometric evaluation in patients with chronic major depression. Biol Psychiatry 2003; 54: 573-83.

42 Karsten J, Nolen WA, Penninx BWJH, Hartman CA. Subthreshold anxiety better defined by symptom self-report than by diagnostic interview. J Affect Disord 2011; 129: 236-43.

43 Epel ES. Psychological and metabolic stress: a recipe for accelerated cellula aging? Hormones 2009; 8: 7-22.

44 Choi J, Fauce SR, Effros RB. Reduced telomerase activity in human T lymphocytes exposed to cortisol. Brain Behav Immun 2008; 22: 600-5.

45 von Zglinicki T. Oxidative stress shortens telomeres. Trends Biochem Sci 2002; 27: 339-44.

46 Damjanovic AK, Yang $\mathrm{Y}$, Glaser $\mathrm{R}$, Kiecolt-Glaser JK, Nguyen $\mathrm{H}$, Laskowski $\mathrm{B}$, et al. Accelerated telomere erosion is associated with a declining immune function of caregivers of Alzheimer's disease patients. J Immunol 2007; 179: 4249-54.

47 Molendijk M, Bus B, Oude Voshaar R, Spinhoven P, Penninx BW, Elzinga B. Serum levels of brain-derived neurotrophic factor in major depressive disorder: state-trait issues, clinical features, and pharmacological treatment. Mol Psychiatry 2010; 16: 1088-95.
48 Vreeburg SA, Hoogendijk WJ, van PJ, Derijk RH, Verhagen JC, van DR, et al. Major depressive disorder and hypothalamic-pituitary-adrenal axis activity: results from a large cohort study. Arch Gen Psychiatry 2009; 66: 617-26.

49 Aviv A, Chen W, Gardner JP, Kimura M, Brimacombe M, Cao X, et al. Leukocyte telomere dynamics: longitudinal findings among young adults in the Bogalusa Heart Study. Am J Epidemiol 2009; 169: 323-9.

50 Nordfjall K, Svenson U, Norrback KF, Adolfsson R, Lenner P, Roos G. The individual blood cell telomere attrition rate is telomere length dependent. PLOS Genet 2009; 5: e1000375.

51 Hoen PW, de Jonge P, Na BY, Farzaneh-Far R, Epel E, Lin J, et al. Depression and leukocyte telomere length in patients with coronary heart disease: data from the Heart and Soul Study. Psychosom Med 2011; 73: 541-7.

52 Shalev I, Moffitt T, Sugden K, Williams B, Houts R, Danese A, et al. Exposure to violence during childhood is associated with telomere erosion from 5 to 10 years of age: a longitudinal study. Mol Psychiatry 2012; 18: 576-81.

53 Puterman E, Lin J, Blackburn E, O'Donovan A, Adler N, Epel E. The power of exercise: buffering the effect of chronic stress on telomere length. PLOS One 2010; 5: e10837.

54 Lin J, Epel E, Blackburn E. Telomeres and lifestyle factors: roles in cellular aging. Mutat Res 2012; 730: 85-9.

55 Osthus IB, Sgura A, Berardinelli F, Alsnes IV, Bronstad E, Rehn T, et al. Telomere length and long-term endurance exercise: does exercise training affect biological age? A pilot study. PLOS One 2012; 7: e52769.

\section{Shabbir Amanullah}

Charlie Brown's best friend Linus has captured the imagination of millions of fans all over the world. No single professional group has probably found as much pleasure in them as those in the field of mental health.

The role of a transitional object is often to provide security, comfort in a world that is very often unpredictable. Even among adults, there are many who still have objects that provide comfort.

The more recent phenomenon of 'constant technology' may be seen as essential for most, but is creating a new generation who find it hard to imagine life without a cell phone. When out of range or charge, a number of parents report their teenage children admit feeling anxious. Constant social media updates with the now ubiquitous 'selfies' are the norm. Little planning or thought goes into it. It is an impulsive need to respond immediately that is creeping in. Technology has created a new reality that requires an alternative to the 'blue blanket'.

Cell phones are no longer just tools of communication. They are lifelines for most of us and increasingly, transitional objects for 'generation cell'. I often wonder, if Linus existed in real life, would he give his blue blanket up for the cell phone? Reality is now arguably in $3 \mathrm{D}-\mathrm{I}$, me, mycellf! 